Wright State University

CORE Scholar

$12-1-1997$

\title{
Spin Splitting of Donor-Bound Excitons in ZnO Due To Combined Stress and Spin Exchange
}

D. C. Reynolds

David C. Look

Wright State University - Main Campus, david.look@wright.edu

B. Jogai

T. C. Collins

Follow this and additional works at: https://corescholar.libraries.wright.edu/physics

Part of the Physics Commons

\section{Repository Citation}

Reynolds, D. C., Look, D. C., Jogai, B., \& Collins, T. C. (1997). Spin Splitting of Donor-Bound Excitons in ZnO Due To Combined Stress and Spin Exchange. Physical Review B, 56 (21), 13753-13756.

https://corescholar.libraries.wright.edu/physics/202

This Article is brought to you for free and open access by the Physics at CORE Scholar. It has been accepted for inclusion in Physics Faculty Publications by an authorized administrator of CORE Scholar. For more information, please contact library-corescholar@wright.edu. 


\title{
ARTICLES
}

\section{Spin splitting of donor-bound excitons in $\mathrm{ZnO}$ due to combined stress and spin exchange}

\author{
D. C. Reynolds, D. C. Look, and B. Jogai \\ University Research Center, Wright State University, Dayton, Ohio 45435 \\ T. C. Collins \\ Oklahoma State University, Whitehurst Hall, Stillwater, Oklahoma 74078
}

(Received 23 June 1997)

\begin{abstract}
There is a marked difference in the growth habits of $\mathrm{ZnO}$ crystals grown from $\mathrm{ZnSe}$ and $\mathrm{ZnS}$ starting materials. Since selenium has a higher boiling point than sulfur and the reaction takes place at lower temperatures when the crystals are grown from $\mathrm{ZnSe}$, the selenium may interact with the growing surface. This may be a cause for residual strain in the $\mathrm{ZnO}$ crystals grown from $\mathrm{ZnSe}$ starting material. The residual strain is observed as spin splittings of the donor-bound exciton transitions. A fourfold splitting is observed due to a combination of strain and spin exchange. [S0163-1829(97)08845-0]
\end{abstract}

\section{INTRODUCTION}

Crystals of $\mathrm{ZnO}$ have been grown from the vapor phase using $\mathrm{ZnS}$ and $\mathrm{ZnSe}$ as the starting materials. The compounds of zinc are heated to sublimation temperature in a flow of argon gas. When the sublimation temperature is reached, oxygen is introduced into the system. The growth habit for $\mathrm{ZnO}$ crystals grown by the reaction of $\mathrm{ZnSe}$ with oxygen differs from of that $\mathrm{ZnO}$ crystals grown from $\mathrm{ZnS}$.

The crystal morphology of $\mathrm{ZnO}$ crystals grown from ZnSe resembles in many respects the crystal morphology reported for ice crystals. ${ }^{1}$ In the case of ice crystals the variation in crystal habit can be attributed to the surface property. This makes the surface diffusion of molecules on the growing face a major factor in determining the crystal habit. This will be strongly influenced by adsorption of impurities on the surface. Considering a (0001) growing surface in a region of moderate supersaturation, molecules arriving at the surface may be incorporated into the surface or migrate over the surface to the edges and be incorporated into the prism faces. It was shown in Ref. 1 that $x_{s}$ (the mean distance a molecule will wander over the surface of the crystal between the time it strikes and the time it is incorporated or evaporates) varies with prism faces and also with temperature. If, on the (0001) growing face of $\mathrm{ZnO}, x_{s}$ is greater than it is for the prism faces, a platelike crystal habit will develop. Molecules arriving on the growing surface will migrate to the prism faces before being incorporated into the lattice. This results in a net transfer of material to the prism faces resulting in a platetype habit with the $c$ axis normal to the plane of the plate. When the crystal dimensions exceed $x_{s}$ the growth is then controlled by the diffusion field. By this time the crystal habit has been established and the diffusion field adjusts to maintain the habit.

The crystal habit for $\mathrm{ZnO}$ crystals grown from a $\mathrm{ZnS}$ reaction with oxygen is appreciably different from those grown from $\mathrm{ZnSe}$. In the platelet-type crystals grown from $\mathrm{ZnS}$, the $c$ axis is contained in the plane of the plate. The mechanism for CdS platelet growth of this type has been reported by
Chikawa and Nakayama ${ }^{2}$ and would be expected to apply to $\mathrm{ZnO}$ as well. The platelets are of very high crystalline quality, containing a very low density of dislocations.

The different crystal morphologies of $\mathrm{ZnO}$ crystals grown from $\mathrm{ZnS}$ and $\mathrm{ZnSe}$ is also reflected in their optical properties. Specifically, strain-induced splitting of the neutraldonor-bound excitons is observed in the crystals grown from $\mathrm{ZnSe}$, while it is absent in the crystals grown from $\mathrm{ZnS}$. The surface diffusion of molecules on the growing surfaces strongly influences the growth habit. The diffusion process is altered by the absorption of foreign atoms or molecules on the surface. Since selenium has a higher boiling point than sulfur and the reaction takes place at lower temperatures when crystals are grown from $\mathrm{ZnSe}$ than is the case for $\mathrm{ZnS}$, the selenium may interact with the growing surface and some may be incorporated in the $\mathrm{ZnO}$ lattice. The incorporated selenium may reside on lattice sites or in interstitial positions. This local defect structure has the potential for producing local strains and also for producing strains in specific crystallographic directions.

At the higher temperature, where the reaction occurs when $\mathrm{ZnS}$ is used as the starting material, there is probably little interaction of the sulfur with the growing $\mathrm{ZnO}$ surface. This would account for the absence of stress-induced spin splitting in these crystals.

\section{EXPERIMENTAL DETAILS}

The details of the growth of the $\mathrm{ZnO}$ crystals used in this experiment were described previously. ${ }^{3}$ The photoluminescence (PL) measurements were made at $2 \mathrm{~K}$ with the sample immersed in liquid He. The spectra were analyzed with a high-resolution, 4-m spectrometer equipped with an RCA C31034A photomultiplier tube for detection. First-order spectra showed no strain splitting in $\mathrm{ZnO}$ crystals grown from either $\mathrm{ZnS}$ or $\mathrm{ZnSe}$ starting materials. However, in second order, strain splitting was observed in $\mathrm{ZnO}$ crystals 


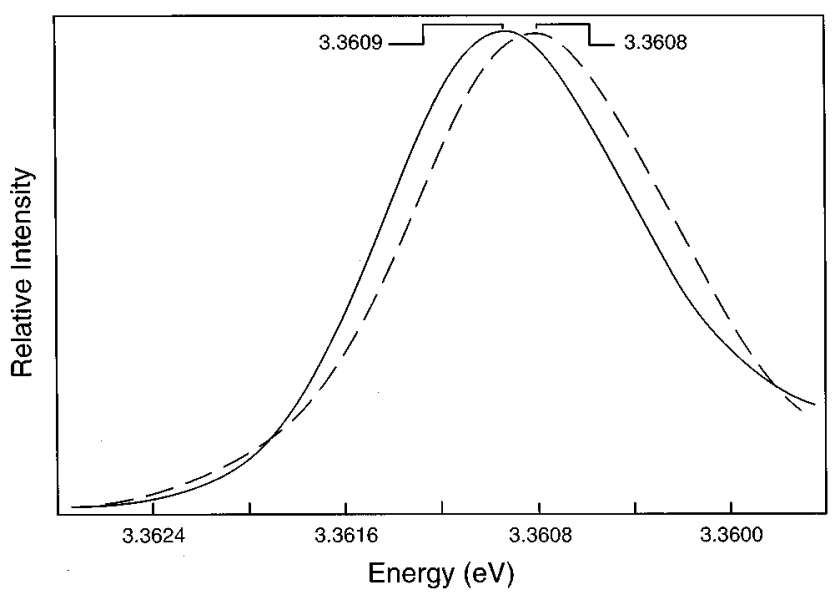

FIG. 1. PL, recorded in first order, from $D^{0}, X$ transitions in two different $\mathrm{ZnO}$ crystals. - grown from $\mathrm{ZnS}$ starting material, --grown from $\mathrm{ZnSe}$ starting material.

grown from $\mathrm{ZnSe}$ starting material but was not observed in $\mathrm{ZnO}$ crystals grown from $\mathrm{ZnS}$ starting material. The PL spectra were excited with the $3250 \AA$ line from a HeCd laser.

\section{EXPERIMENTAL RESULTS AND DISCUSSION}

The splitting of the exciton lines in wurtzite crystals when exposed to an applied stress having a particular orientation with respect to the crystallographic axes was reported by Koda and Langer. ${ }^{4}$ In the case of wurtzite crystals all of the orbital degeneracies of the valence band are lifted by the trigonal crystal field and spin-orbit interaction. The above phenomena could not be explained by the one-electron band scheme and deformation potential theory. A theoretical interpretation was provided by Akimoto and Hasegawa. ${ }^{5}$ They found that the combined effects of stress and the electronhole exchange interaction in a quasicubic model were able to predict the splitting and polarization pattern of the free exciton. These studies were extended to several other materials by Langer et al. ${ }^{6}$ The stress effects on excitons bound to shallow acceptors in zinc-blende materials were later investigated. ${ }^{7,8}$ This is a report of spin splitting due to stress in donor-bound excitons.

The donor-bound exciton $\left(D^{0}, X\right)$ transitions for two different $\mathrm{ZnO}$ crystals are shown in Fig. 1. The solid line shows the transition from a crystal grown from $\mathrm{ZnS}$ starting mate-

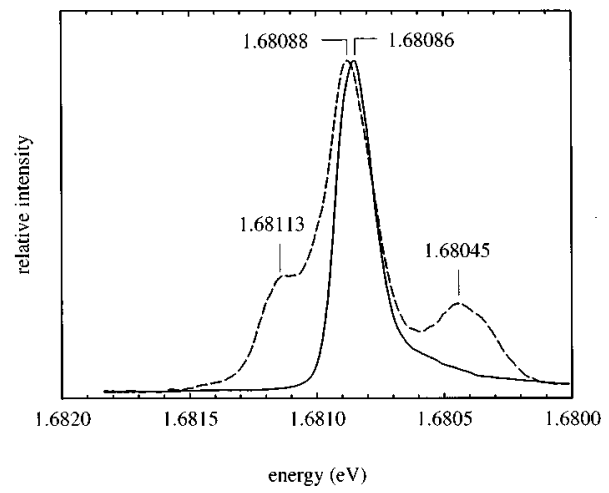

FIG. 2. Same as Fig. 1 except recorded in second order.
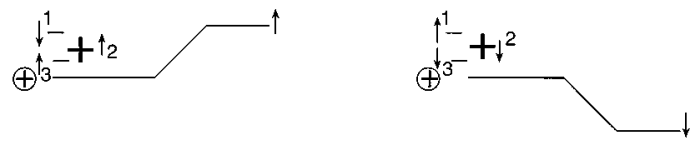

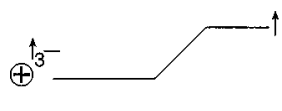

(a)

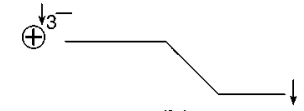

(b)
FIG. 3. Spin configurations for $D^{0}, X$ complexes.

rial, while the dashed line shows the transition from a $\mathrm{ZnO}$ crystal grown from $\mathrm{ZnSe}$ starting material. Both transitions were recorded in first order. The same spectra, but in second order, are shown in Fig. 2. The solid curve is for the sample grown from $\mathrm{ZnS}$, the dashed curve is for the sample grown from $\mathrm{ZnSe}$. This illustrates the advantage of second-order spectra in resolving spectral features. The resolving power $R$ of a grating is defined as the minimum separation of two spectral features for which they appear distinct. The resolving power is expressed as

$$
R=\lambda / d \lambda=n N,
$$

where $n$ is the grating order and $N$ is the number of ruled lines. The expression shows that the resolving power is increased by a factor of 2 in second order. Spin splitting is clearly resolved in the dashed curve, which results from the combined effects of stress and the exchange interaction. The $D^{0}, X$ transition is shown schematically in Fig. 3. In the upper state the two electron spins pair to give a bonding state. When the exciton collapses, the final state is the neutral donor, either in ground state or an excited state. Considering electron-hole exchange interaction for the exciton in the upper state, the electron and hole spins will pair. The electron and hole spins are diagrammatically depicted in Fig. 3. In Fig. 3(a), the hole spin associated with the exciton is up $\uparrow 2$; the electron spin associated with the exciton is down $\downarrow 1$. Therefore, the electron spin associated with the donor will be up $\uparrow 3$. If the exciton collapses from this spin configuration,

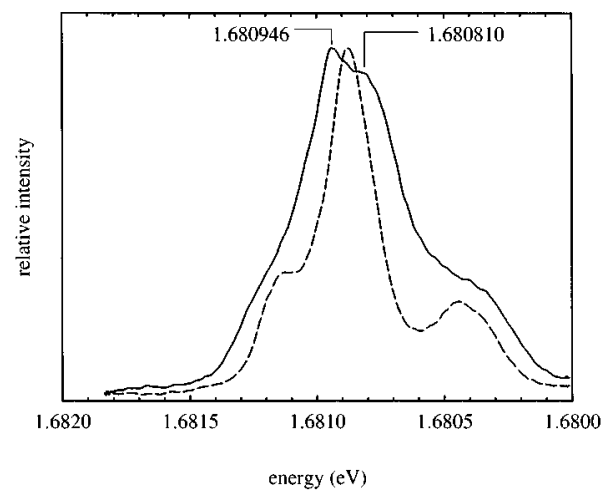

FIG. 4. Fourfold stress-induced spin splitting in $D^{0}, X$ transitions in $\mathrm{ZnO}$ grown from $\mathrm{ZnSe}$ starting material. - applied magnetic field of $36 \mathrm{kG}$, --- zero magnetic field. 


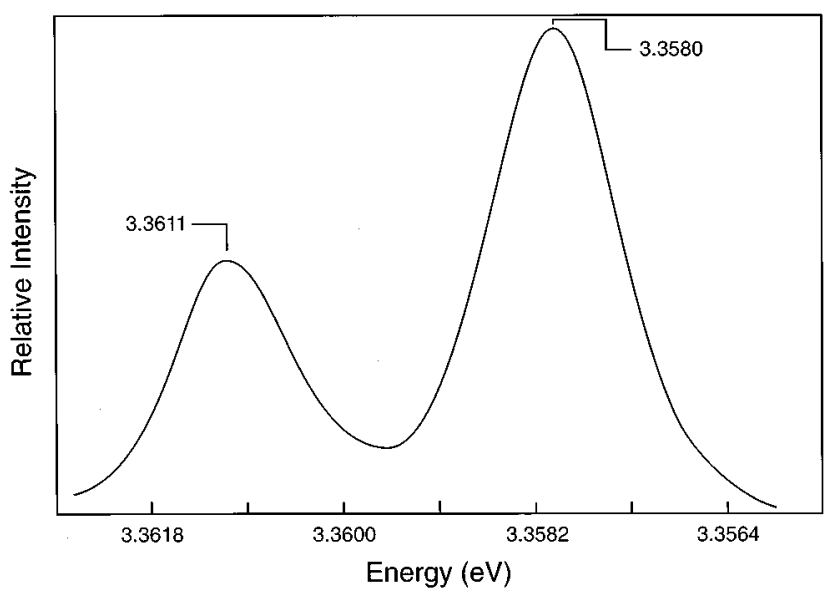

FIG. 5. PL, recorded in first order, from a second sample of $\mathrm{ZnO}$ grown from $\mathrm{ZnSe}$ starting material. Two $D^{0}, X$ complexes are present in this sample.

the final state will have an electron spin that is up $\uparrow 3$. When the spins exchange as in Fig. 3(b), spin 2 will be down $\downarrow 2$; spin 1 will be up $\uparrow 1$. Therefore, spin 3 will be down $\downarrow 3$. When the exciton collapses from this spin configuration, the final state will have an electron spin that is down $\downarrow 3$. This results in a stress splitting of both the initial and final state of the transition. The splitting of the ground state shown in Fig. 3 can be explained by using the theory outlined in Ref. 6 . If one takes the electron spin of the donor-bound electron being aligned or antialigned to that of the charge cloud of the donor, there is a strain matrix element which splits into two states. In the case of the wurtzite lattice, the magnitude of the splitting will also be directionally dependent. Thus one expects a different magnitude of splitting due to the orientation of the strain field.

Therefore, one would expect to observe a fourfold spin splitting due to stress. Three lines are clearly observed and when an external magnetic field is applied, a further splitting of the high intensity transition is observed. In the absence of a magnetic field, these two transitions have essentially the same energy. This is shown in Fig. 4 where the dashed curve shows the transitions in zero magnetic field, while the solid curve shows the transitions in an applied field of $36 \mathrm{kG}$. These are the spin-conserving transitions and will have higher intensity than the spin-flip transitions.

In this growth habit the $c$ axis is normal to the plane of the plate and the magnetic-field direction in our system is

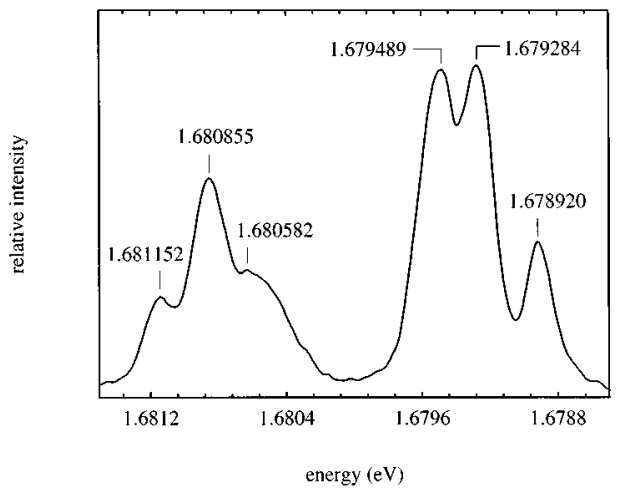

FIG. 6. Same as Fig. 5 except recorded in second order.

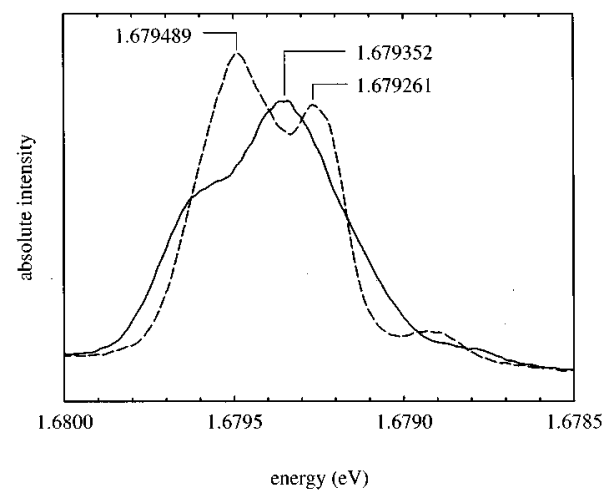

FIG. 7. PL for the $D^{0}, X$ transition at $3.3580 \mathrm{eV}$ in Fig. 5 in zero field ---, and in an applied field of $36 \mathrm{kG}$.

therefore normal to the $c$ axis of the crystal. The hole $g$ value goes as $\left(g_{h}=g_{h \|} \cos \theta\right)$, where $\theta$ is the angle between the $c$ axis of the crystal and the magnetic-field direction. In this case $\theta=90^{\circ}$; therefore, the hole $g$ value in the initial state, shown in Fig. 3, will go to zero. The electron in the final state has an isotropic $g$ value and the observed splittings, due to the applied magnetic field, are due to electron-spin splitting.

Another $\mathrm{ZnO}$ crystal, which was also grown from $\mathrm{ZnSe}$ starting material, was investigated. The $D^{0}, X$ transitions from this sample recorded in first order, are shown in Fig. 5. The high-energy transition is the same $D^{0}, X$ transition as was observed from the sample shown in Fig. 1. The lower energy $D^{0}, X$ transition is due to another donor. These two transitions when recorded in second order appear as shown in Fig. 6. The higher-energy transition is essentially a duplicate of the same $D^{0}, X$ transition from the sample shown in Fig. 2. The lower energy $D^{0}, X$ transition shows a different spin splitting; the two spin-conserving transitions are separated in energy. When an external magnetic field is applied, these two transitions move toward each other as shown in Fig. 7. The dashed curve shows the zero-field case, while the solid curve was recorded with an applied field of $36 \mathrm{kG}$.

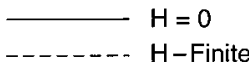

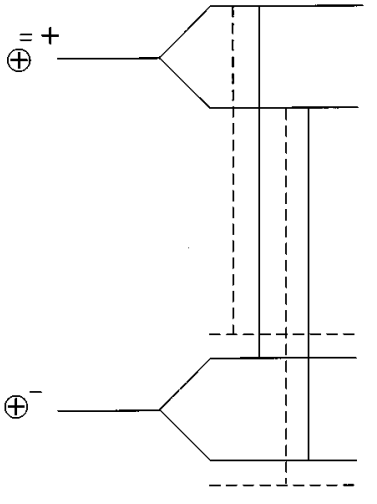

(a)

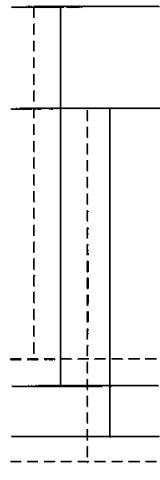

(b)
FIG. 8. Model to explain the magnetic-field splittings of the two $D^{0}, X$ transitions shown in Fig. 5 . 


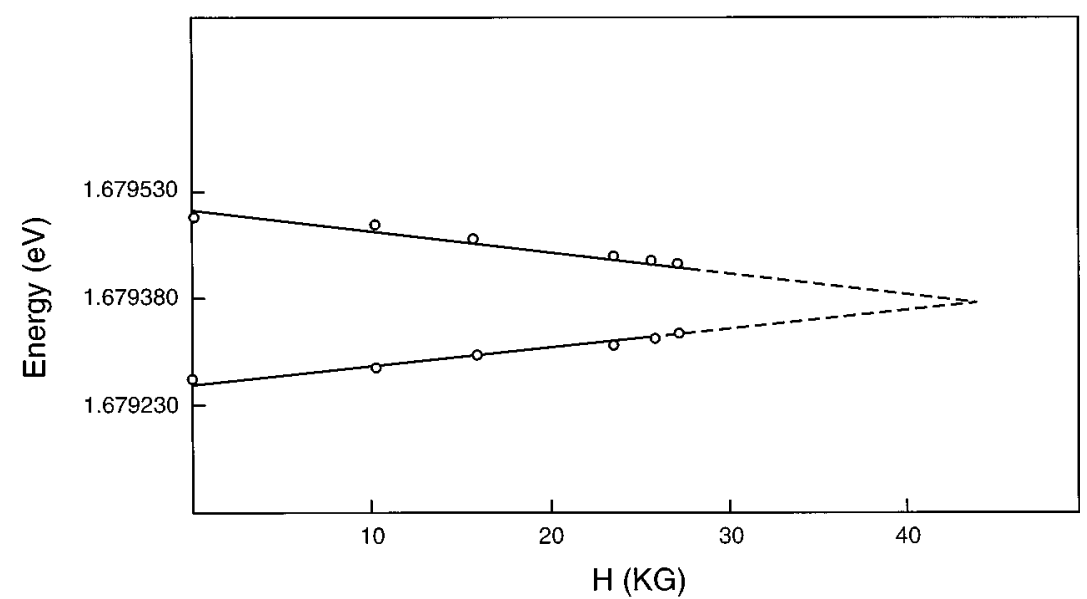

FIG. 9. Magnetic-field splitting as a function of applied field for the $D^{0}, X$ transition shown in Fig. 7 .

The different behavior of these two $D^{0}, X$ transitions can be understood from the schematic diagram in Fig. 8. Focusing on Fig. 8(a), the stress-induced spin splittings of the initial and final state are essentially the same. From this splitting it is seen that the two spin-conserving transitions (solid lines) have essentially the same energy.

When an external magnetic field is applied, we showed above that the hole $g$ value in the initial state goes to zero, resulting in zero magnetic field splitting of this state. The electron in the final state will show additional spin splitting with applied magnetic field. The additional splitting will result in lower energy for one of the spin-conserving transitions and an increase in energy for the other. This will cause the two spin-conserving transitions that are nearly equal in energy to move apart in energy with applied magnetic field as shown in Fig. 4.

Turning to Fig. 8(b), if the initial state has a larger spin splitting than the final state, then one of the spin-conserving transitions will have a larger energy than the other (solid lines), as seen in Fig. 6. When an external magnetic field is applied, again there will be no magnetic-field splitting of the initial state but there will be an additional electron-spin splitting in the final state. The higher-energy spin conserving transition will move to lower energy, while the lower energy spin conserving transition will move toward higher energy (dashed lines). This will result in the two spin conserving transitions moving towards each other in energy as shown in Fig. 7.

It is believed that the difference in stress-induced spin splitting for the two donor-bound excitons in the second sample is due to the stress being oriented in different crys- tallographic directions. One would expect the magnitude of the stress-induced spin splitting would be dependent on the crystallographic orientation of the stress.

It was pointed out above that the energy separation of the spin-conserving transitions, shown in Fig. 7, decreases with applied magnetic field. The total magnetic-field splitting is associated with the electron-spin splitting in the final state as shown in Fig. 8. From this splitting one can obtain a reasonable $g$ value for the electron. The lines are broader than would be desired for a precise determination. The magneticfield splitting as a function of applied magnetic field is plotted in Fig. 9. A linear splitting is observed which is extrapolated to zero splitting. This determines the applied field necessary to bring the lines together, which would otherwise be difficult, due to the line width. Using a magnetic field of $44 \mathrm{kG}$ and the measured line separation at zero field, an electron $g$ value $g_{e}=1.96$ was determined. This agrees quite well with the previously reported electron $g$ value, 1.95 , determined from a different donor-bound exciton transition in $\mathrm{ZnO}^{9}$

In conclusion a fourfold spin splitting is observed in donor-bound exciton transitions in $\mathrm{ZnO}$ crystals grown from $\mathrm{ZnSe}$ starting material. This splitting is caused by a combination of strain and spin exchange.

\section{ACKNOWLEDGMENTS}

The authors express their sincere gratitude to C. Huang and C. W. Litton for technical support. This work was performed at Wright Laboratory, Avionics Directorate (WL/ AADP), Wright Patterson Air Force Base under USAF Contract No. F33615-95-C-1619. This work was partially supported by AFOSR.
${ }^{1}$ B. J. Mason, The Art and Science of Growing Crystals (Wiley, New York, 1963), pp. 119-150.

${ }^{2}$ J. Chikawa and T. Nakayama, J. Appl. Phys. 35, 2493 (1964).

${ }^{3}$ Y. S. Park and D. C. Reynolds, J. Appl. Phys. 38, 756 (1967).

${ }^{4}$ T. Koda and D. W. Langer, Phys. Rev. Lett. 20, 50 (1958).

${ }^{5}$ O. Akimoto and H. Hasegawa, Phys. Rev. Lett. 20, 916 (1968).

${ }^{6}$ D. W. Langer, R. N. Euwema, K. Era, and T. Koda, Phys. Rev. 2,
4005 (1970).

${ }^{7}$ M. Schmidt, T. N. Morgan, and W. Schairer, Phys. Rev. 11, 5002 (1975).

${ }^{8}$ H. Mathieu, J. Camassel, and B. Chekroun, Phys. Rev. 29, 3438 (1984).

${ }^{9}$ D. C. Reynolds and T. C. Collins, Phys. Rev. 185, 1099 (1969). 